Final Report on Scientific Facilities Initiative Contract ER45629

\title{
DEVELOPMENT OF NEXT-GENERATION DETECTORS AND INSTRUMENTATION FOR PHOTOELECTRON SPECTROSCOPY, DIFFRACTION AND HOLOGRAPHY
}

\author{
Principal investigator: Charles S. Fadley \\ Department of Physics, University of California Davis
}

For reference in discussing the achievements under this SFI funding, the abstract of the proposal is repeated below:

"We will develop new multichannel detectors for use in photoelectron spectroscopy (as well as other types of high-count-rate spectroscopy) that will operate at rates of up to $1 \mathrm{GHz}$. Such detectors are crucial to the full utilization of the high-brightness radiation generated by the ALS and other thirdgeneration synchrotron radiation sources, and an industrial partnership to commercialize them is also underway.

In addition, new software and hardware will be developed to permit rapidly and accurately scanning photoelectron spectra that will be accumulated in as little as a msec.

A versatile next-generation sample goniometer permitting equally rapid scanning of specimen angles or photon energies for angle-resolved photoemission studies, photoelectron diffraction, and photoelectron holography measurements, will also be designed and constructed.

These capabilities will be incorporated into a unique photoelectron spectrometer/diffractometer at the ALS which includes ultrahigh energy resolution, in situ rotation, variable polarization, and optional spin detection.

This overall system will be used in studies of a variety of problems including magnetic metals and oxides; semiconductors in interaction with metals and halogens; metal/metal, metal/semiconductor, and metal-oxide/metal-oxide multilayers; systems exhibiting giant and colossal magnetoresistance; and high Tc materials."

We will review progress relative to each of the paragraphs above.

-High-speed multichannel detector development:

We have designed, constructed, and carried out successful tests of a first prototype multichannel detector that has demonstrated linear counting rate capability up to $1 \mathrm{GHz}$, spatial resolution of $\mathbf{7 5}$ microns, and the ability to accumulate spectra at the ALS (see addendum to Appendix 1).

This successful development has led to a current ALS-funded project to take the lessons learned from the prototype and construct a more robust and generally useful second generation detector. This detector is just now in final tests, and will be installed in the Fadley spectrometer at the ALS in April, 2003. This detector will count at rates 100-1000x faster than anything currently available, and various research groups and synchrotron radiation facilities around the world have expressed interest in having copies of it. There is as well continuing interest from Quantar Technology in a commercial version of it.

These detector developments are described in the attached Appendix 1.

Beyond these specific detectors, we have been active in promoting more generally the need for advanced detectors for synchrotron radiation research, and this led first to a dedicated workshop in Washington, then to the coordinating DetectorSync working group, and finally to a dedicated effort to fund such activities within DOE, headed by Pedro Montano. 
-Parallel software and hardware development:

Taking advantage of both source brightness and these enhanced detection capabilities has also led to the writing of a very flexible operating system for the Scienta SES200 electron spectrometer that is the principal unit in the Advanced Photoelectron Spectrometer/Diffractometer (APSD) that has been constructed by the Fadley group over the past decade. The APSD is presently situated on an elliptically-polarized undulator at the ALS (beamline 4.0.2). This operating system is written in LabWindows/CVI, and it includes the ability to control data acquisition with the existing Scienta detector, the new high-speed multichannel detector, and also a microMott spin detector. Spectra can be accumulated in "snapshot" mode in as little as 100 microseconds with the high-speed detector and this software, with further time-resolved measurements planned for the near future.

- Next-generation sample goniometers and sample handling capabilities:

The sample goniometer for the APSD has also been improved so as to permit reliably cooling down to $130 \mathrm{~K}$, and heating up to $2000 \mathrm{~K}$. A loadlock was also installed on the APSD system to permit the rapid introduction and removal of samples. Within this loadlock, a system for cleaving single crystal metal oxide samples was also designed and installed. This cleaver has been used for an extensive spectroscopic investigation of singles crystals of colossal magnetoresistive (CMR) oxides.

SFI funds were also used to initiate the design and construction of a totally new sample goniometer that will expand the temperature range from ca. $10 \mathrm{~K}$ to $2000 \mathrm{~K}$, with complete rotational flexibility and rapid scanning capability. The latter was subsequently funded through the Materials Sciences Division of LBNL and is scheduled for completion in summer, 2003. No equivalent sample goniometer is available from commercial sources.

-Incorporation of instrumentation improvements into a unique experimental system:

The high-speed detector, operating system, and other hardware improvements mentioned above have all been incorporated into the previously-mentioned Advanced Photoelectron Spectrometer/Diffractometer. This system is pictured on the next page, with highlighted items being the direct result of SFI support. The reduction of the final SFI budget by approximately 1/2 from that requested (from ca. $\$ 800 \mathrm{k}$ to ca. $\$ 400 \mathrm{k}$ ) inevitably slowed down certain items, but everything proposed is now nearing either completed or nearing completion.

To our knowledge the resulting APSD system provides a set of capabilities unique in the world for soft X-ray synchrotron radiation spectroscopy. It is noteworthy here that, stimulated by the SFI award, followon funding via LBNL and UC Davis totally about \$250k was obtained for a Scienta soft $x$-ray emission spectrometer. This spectrometer has now been installed in the system, with the overall result that all of the relevant soft $x$-ray spectroscopies (core and valence photoemission, x-ray absorption, and x-ray emission/x-ray inelastic scattering) can be carried out on a single sample. This capability has first been utilized in our study of some CMR oxides.

- Specific scientific studies and publications using the new instrumentation:

The publications list below indicates various specific research projects completed with this instrumentation. In brief, the areas, and relevant publications, as numbered in this list, are:

--Metal, metal oxide, semiconductor, and adsorbate surface structures via photoelectron diffraction, including new algorithms for data analysis: papers 1, 3, 9, 10, 11, 12, 13, 14, 24

--Further development of photoelectron holography as a new method for near-surface atomic structure determinations: papers 23 and 29

--First measurements of reaction kinetics using time-resolved photoelectron spectroscopy: paper 14

--First exploration and theoretical explanation of multi-atom resonant photoemission: papers $2,6,8,15,17,25,28,34$

--Observation of a elevated surface magnetic phase transition temperature for Gd(0001), via both core multiplet intensities (paper 4) and energy splittings (paper 32), and associated theoretical interpretations of these effects (papers 21, 22, 32) 
--Studies of circular dichroism in core photoelectron emission from non-magnetic materials: papers 5, 16

--Studies of magnetic circular dichroism in core photoelectron emission from a ferromagnet (Gd), including advances in theoretical interpretation: papers 7, 18, 26, 30

--Development of a new soft $x$-ray standing-wave method for probing buried interfaces, with first application to the GMR interface Fe/Cr: papers 20, 27, 31

--Observation of a previously inobserved high-temperature electronic phase in the colossal magnetoresistive oxides $\mathrm{La}_{1-x} \mathrm{Sr}_{x} \mathrm{MnO}_{3}(x=0.3,0.4)$, with implications for polaron formation and phase separation: paper 33.

Publications directly resulting from the instrumentation developed under this SFI contract:

(1) "The Structure of Formate on $\mathrm{TiO}_{2}(110)$ from Scanned-Energy and Scanned-Angle Photoelectron Diffraction", S. Thevuthasan, G.S. Herman, Y.J. Kim, S.A. Chambers, C.H.F. Peden, Z. Wang, R. Ynzunza, E.D. Tober, J. Morais, and C.S. Fadley, Surf. Sci. 401,261 (1998),

(2) "Multi-Atom Resonant Photoemission: A Tool for Determining Near-Neighbor Atomic Identities and Bonding", A. Kay, E. Arenholz, S. Mun, J. Garcia de Abajo, C.S. Fadley, R. Denecke, Z. Hussain, and M.A. Van Hove, Science 281, 679 (1998). ). [See later publication, Phys. Rev. B $\underline{63}, 5119$ (2001), for update of this work.]

(3) "Direct Structural Analysis of (1x1)-(1x12) Oxygen on W(110) by Full-Solid-Angle X-Ray Photoelectron Diffraction with Chemical State Resolution", H. Daimon, R. Ynzunza, J. Palomares, H. Takagi, and C.S. Fadley, Surf. Sci. 408, 260 (1998).

(4) "Observation of a Ferromagnetic-to-Paramagnetic Transition on a Ferromagnetic Surface Using Spin-Polarized Photoelectron Diffraction: Gd(0001)", E.D. Tober, F. J. Palomares, R. X. Ynzunza, R. Denecke, J. Morais Z. Wang, G. Biino, J. Liesegang, Z. Hussain, and C.S. Fadley, Phys. Rev. Lett. 81, 2360 (1998).

(5) "Circular Dichroism in Core-Level Emission from O/W(110): Experiment and Theory", $H$. Daimon, R.X. Ynzunza, F.J. Palomares, E.D. Tober, Z.X. Wang, A.P. Kaduwela, M.A. Van Hove, and C.S. Fadley, Phys. Rev B $\underline{58}, 9662$ (1998).

(6) "Interatomic Multi-Atom Resonant Photoemission: Theory and Systematics", F.J. Garcia de Abajo, C.S. Fadley, and M.A. Van Hove, Phys. Rev. Letters 82, 4126 (1999).

(7) "Magnetic Circular Dichroism in Photoelectron Angular Distributions from Gd(0001)", R. Denecke, J. Morais, R.X. Ynzunza, J.G. Menchero, J. Liesegang, and C.S. Fadley, J. Electron Spectrosc. 101-103, 263 (1999).

(8) "Multiple Atom Resonant Photoemission" A New Technique for Studying Near-Neighbor Atomic Identities and Bonding", A.W. Kay, E. Arenholz, B.S. Mun, J. Garcia de Abajo, C.S. Fadley, R. Denecke, Z. Hussain, and M.A. Van Hove, J. Electron Spectrosc.101-103, 647 (1999).

(9) "Surface Structure of MBE-Grown $\alpha-\mathrm{Fe}_{2} \mathrm{O}_{3}(0001)$ by Intermediate-Energy X-ray Photoelectron Diffraction", S. Thevuthasan, Y.J. Kim, S.I. Yi, S.A. Chambers, J. Morais, R. Denecke, C.S. Fadley, P. Liu, T. Kendelewicz, and G.E. Brown, Jr., Surf. Sci. 425,:276 (1999).

(10) "Atomic and Electronic-Band Structures of Anomalous Carbon Dimers on 3C-SiC(001)c(2x2)", H.W. Yeom, M. Shimomura, J. Kitamura, S. Hara, K. Tono, I. Matsuda, B.S. Mun, W.A.R. Huff, S. Kono, T. Ohta, S. Yoshida, H. Okushi, K. Kajimura, and C.S. Fadley, Phys. Rev. Lett. 83, 1640-1643 (1999).

(11) "Full-Solid-Angle Photoelectron Diffraction from Bulk and Surface Atoms of Clean W(110)", R.X. Ynzunza, E.D. Tober, F.J. Palomares, Z. Wang, H. Daimon, Z. Hussain, M.A. Van Hove, and C.S. Fadley, Surf. Sci. 441, 301-310 (1999). 
(12) "Structure Determination for Saturated (1x1) Oxygen on W(110) from Full-Solid-Angle Photoelectron Diffraction with Chemical-State Resolution", R. Ynzunza, H. Daimon, J. Palomares, E.D. Tober, Z. Wang, Y. Chen, M.A. Van Hove, and C.S. Fadley, Surf. Sci., 442, $27-$ 35 (1999).

(13) "Surface Core-Level-Shift Photoelectron Diffraction Study of the $\beta$-SiC(001)-c(2x2) Surface", M. Shimomura, H.W. Yeom, B.S. Mun, C.S. Fadley, S. Hara, S. Yoshida, and S. Kono, Surf. Sci. 438, 237-241 (1999).

(14) " Kinetics and Atomic Structure of Oxygen Adsorption on W(110) from Time- and StateResolved Photoelectron Spectroscopy and Full-Solid-Angle Photoelectron Diffraction", R.X. Ynzunza, F.J. Palomares, E.D. Tober, Z. Wang, H. Daimon, Y. Chen, Z. Hussain, J. Liesegang, M.A. VanHove, and C.S. Fadley, Surf. Sci. $\underline{459}, 69$ (2000).

(15) "Multi-Atom Resonant Photoemission", C.S. Fadley, E. Arenholz, A.W. Kay, J. Garcia de Abajo, B.S. Mun, S.-H. Yang, Z. Hussain, and M.A. Van Hove, in X-ray and Inner Shell Processes, AIP Conference Proceedings No. 506. R.W. Dunford et al., Eds. (AIP, New York, 2000), pp. 251-272.

(16) "Circular Dichroism in Core Photoelectron Emission from (1x1) Oxygen on W(110): Experiment and Multiple-Scattering Theory", R.X. Ynzunza, H. Daimon, F.J.Palomares, E.D.Tober, Z. Wang, F.J. Garcia de Abajo, J. Morais, R. Denecke, J.B. Kortright, Z. Hussain, M.A. Van Hove, and C.S. Fadley, J. Electron Spectrosc. 106, 7 (2000).

(17) "Observation of Multi-Atom Resonant Photoemission via Secondary Processes: Auger Decay and X-ray Fluorescence", E. Arenholz, A. W. Kay, C. S. Fadley, M. M. Grush, T. A. Callcott, D. L. Ederer, C. Heske, and Z. Hussain, Phys. Rev. B 61, 7183 (2000). [See Phys. Rev. $B \underline{63}, 115119$ (2001) for update of this work.]

(18) "Magnetic Dichroism in Core-Level Photoemission from Gd(0001)", J. Morais, G.H. Fecher, R. Denecke, Z. Hussain, and C.S. Fadley, J. Appl. Phys. 874900 (2000).

(19) "Observation of the Two-Hole Satellite in $\mathrm{Cr}$ and Fe Metal by Resonant Photoemission at the $2 p$ Absorption Energy", S. Hüfner, S.-H. Yang, B. S. Mun, C.S. Fadley, J. Schäfer, E. Rotenberg, and S. D. Kevan, Phys. Rev. B $\underline{61}, 12582$ (2000).

(20) "Depth-Resolved Photoemission Spectroscopy from Surfaces and Buried Layers with Soft X-Ray Standing Waves", S.-H. Yang, B.S. Mun, A.W. Kay, S.-K. Kim, J.B. Kortright, J.H. Underwood, Z. Hussain, and C.S. Fadley, Surf. Sci. Lett. 461, L557 (2000).

(21) "Electron Correlation Effects at the Gd(0001) Surface", A.B. Shick, W.E. Pickett, and C.S. Fadley, J. Appl. Phys. 87, 5878 (2000).

(22) "Electron Correlation Effects and Magnetic Ordering at the Gd(0001) Surface", A.B. Shick, W.E. Pickett, and C.S. Fadley, Phys. Rev. B, Rapid Comm. 61, R9213 (2000).

(23) "Photoelectron Holography Analysis of the W(110)(1 x 1)-O Surface", H. Takagi, H. Daimon, J. Palomares, C.S. Fadley, Surf. Sci. $\underline{470}, 189$ (2001).

(24) "Multiple Scattering of Electrons in Solids and Molecules: A Novel Cluster Approach", F.J.Garcia de Abajo, M.A. Van Hove, and C.S. Fadley, Phys. Rev. B $\underline{63}, 075404$ (2001).

(25) "Multi-Atom Resonant Photoemission", A.W. Kay, F.J. Garcia de Abajo, S.-H. Yang, E. Arenholz, B.S. Mun, N. Mannella, Z. Hussain, M.A. Van Hove, and C.S. Fadley, Phys. Rev. B $\underline{63}$, 115119 (2001).

(26) "Dichroism in angular resolved XPS from gadolinium core-levels", J. Morais, G. Fecher, R. Denecke, J. Liesegang, C.S. Fadley, J. Electron Spectrosc. 114, 783 (2001). 
(27) "Depth-resolved photoemission spectroscopy with soft x-ray standing waves", S.-H. Yang, B.S. Mun, A.W. Kay, S.K. Kim, J.B. Kortright, J.H. Underwood, Z. Hussain, C.S. Fadley, J. Electron Spectrosc. 114, 1089 (2001).

(28) "Multi-atom resonant photoemission", A.W. Kay, F.J. Garcia de Abajo, S.-H. Yang, E. Arenholz, B.S. Mun, N. Mannella, Z. Hussain, M.A. Van Hove, and C.S. Fadley, J. Electron Spectrosc. 114, 1179 (2001).

(29) I "Photoelectron and X-ray Holography by Contrast: Enhancing Image Quality and Dimensionality", C.S. Fadley, M.A. Van Hove, A. Kaduwela, S. Omori, L. Zhao, and S. Marchesini, J. Phys. Cond. Mat. 13, 10517 (2001).

(30) “Angular and Temperature Dependence of Magnetic Circular Dichroism in 4d Core-Level Photoemission from Gd(0001)", R. Denecke, J. Morais, R.X. Ynzunza, G. Fecher, J.G. Menchero, J. Liesegang, J.B. Kortright, Z. Hussain, and C.S. Fadley, Phys. Rev. B $\underline{65}, 245421$ (2002).

(31) "Probing Buried Interfaces with Soft X-ray Standing Wave Spectroscopy: Application to the Fe/Cr Interface", S.-H. Yang, B.S. Mun, N. Mannella, S.-K. Kim, J.B. Kortright, J. Underwood, F. Salmassi, E. Arenholz, A. Young, Z. Hussain, M.A. Van Hove, and C.S. Fadley, J. Phys. Cond. Matt. 14, L406 (2002).

Submitted and in preparation:

(32) "Observation of Magnetic Transitions via Core-Level Multiplet Energy Separations: Gd(0001)", E.D. Tober, F.J. Palomares, R.X. Ynzunza, R. Denecke, J. Morais, Z. Wang, G. Biino, J. Liesegang, Z. Hussain, A.Shick, W. Pickett, and C.S. Fadley, Phys. Rev.Lett., submitted.

(33) "Observation of a High-Temperature Electronic Phase Transition and Polaron Formation in the Colossal Magnetoresistive Oxides $\mathrm{La}_{1-\mathrm{x}} \mathrm{Sr}_{x} \mathrm{MnO}_{3}(\mathrm{x}=0.3,0.4) ", \mathrm{~N}$. Mannella, A. Rosenhahn, S. Marchesini, B.S. Mun, S.-H. Yang, J. Guo, M.W. West, A. Mei, Y. Tomioka, Y. Tokura, K. Ibrahim, E. Arenholz, A. Young, M. A. Van Hove and C.S. Fadley, to be submitted to Science.

(34) "Multi-Atom Resonance and X-ray Optical Effects in Photoemission from NiO", N. Mannella, S.-H. Yang, B.S. Mun, L. Zhao, A.T. Young, E. Arenholz, and C.S. Fadley, to be submitted to Phys. Rev. B, Rapid Comm. 


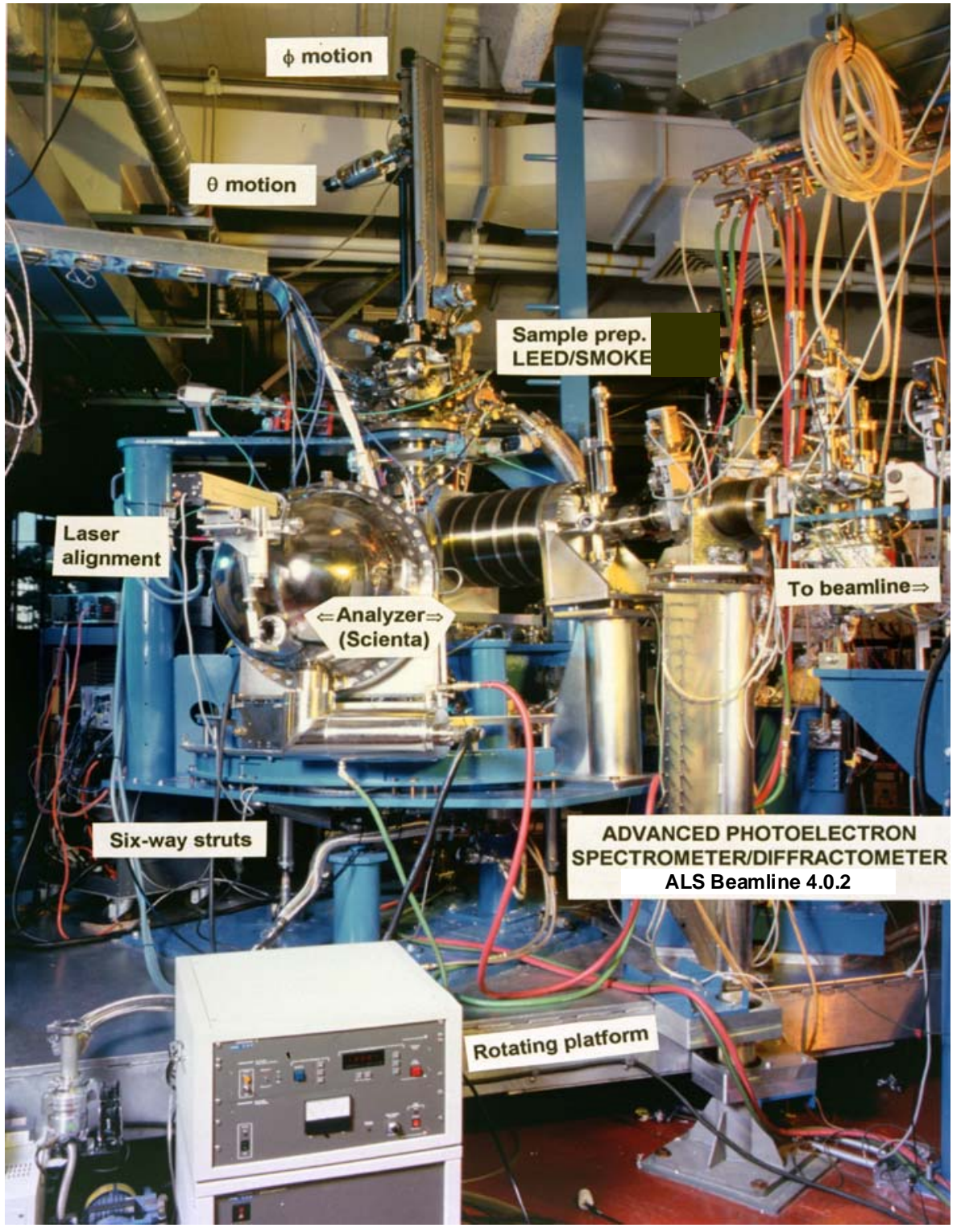

\section{ADVANCED PHOTOELECTRON SPECTROMETER/ \\ DIFFRACTOMETER (APSD)}

-Highest brightness radiation from variable-polarization ALS undulator (EPU 4.0.2) $\leftrightarrow O \wedge O$

-Rotateable high-resolution, highthroughput electron spectrometer (Scienta SES 200)

-High-precision custom sample goniometer, 150K-2300K (SFI)

-Versatile sample preparation \& characterization: loadlock, crystal cleavage, Knudsen cells, LEED, in situ magnetization,....(SFI)

-Rotateable high-resolution soft $x$ ray spectrometer (Scienta XES 300)

\section{Improvements in progress:}

- GHz-rate ultrahigh-speed multichannel detector (early '03) (SFI)

-Low-T sample goniometer, full $\theta, \phi$ rotation, 10K-2300K (mid '03) (SFI)

-MicroMott spin detector (mid '03) (SFI)

-Refocussing mirror $\rightarrow 3-5 x$ higher intensities ('03) 


\section{Appendix 1 \\ Ultrahigh-Speed, One-Dimensional Detector Array for Synchrotron Radiation Experiments \\ (Advanced Light Source, LBNL, May 2002)}

An ultrahigh-speed ( 2 GHz), one-dimensional detector array for electrons and vuv/x-ray photons is under development at ALS/LBNL. This advanced detector should be capable of handling the highest count rates encountered at third-generation synchrotron radiation sources, thus permitting the fullest utilization of the radiation, as well as new kinds of time-resolved experiments. The detector is also free from non-linearity problems that have been encountered in the past with CCD detectors, and it pushes the upper end of linear counting upward by a factor of approximately 100 .

Electrons or photons are detected and counted by a state-of-the-art configuration of microchannel plates, a finely spaced array of charge collection electrodes, and custom-designed integrated circuits. The electrons impinge on a pair of multichannel plates in a chevron configuration. The active counting area is $38.4 \mathrm{~mm}$ by $10 \mathrm{~mm}$. The charge clouds emitted from the backside of the channel plate are collected on 768 collection strips with 50 micron spacing that are wire bonded to 6 pairs of signal processing ICs. Each frontend IC (the CAFE-M, a proven device from high energy physics) has 128 channels of amplifiers (peaking time $25 \mathrm{~ns}$ ) and discriminators. The pulse-pair resolution is $50 \mathrm{~ns}$ and this leads to a maximum linear countrate/channel of $2 \mathrm{MHz}$. The frontend IC is followed by a custom-designed buffered multichannel counter (BMC) IC permitting readout with no deadtime. The BMC IC features a 24-bit buffered counter for each channel, programmable control of CAFE-M threshold levels and calibration signals, and a serial link for the transfer of commands and data.

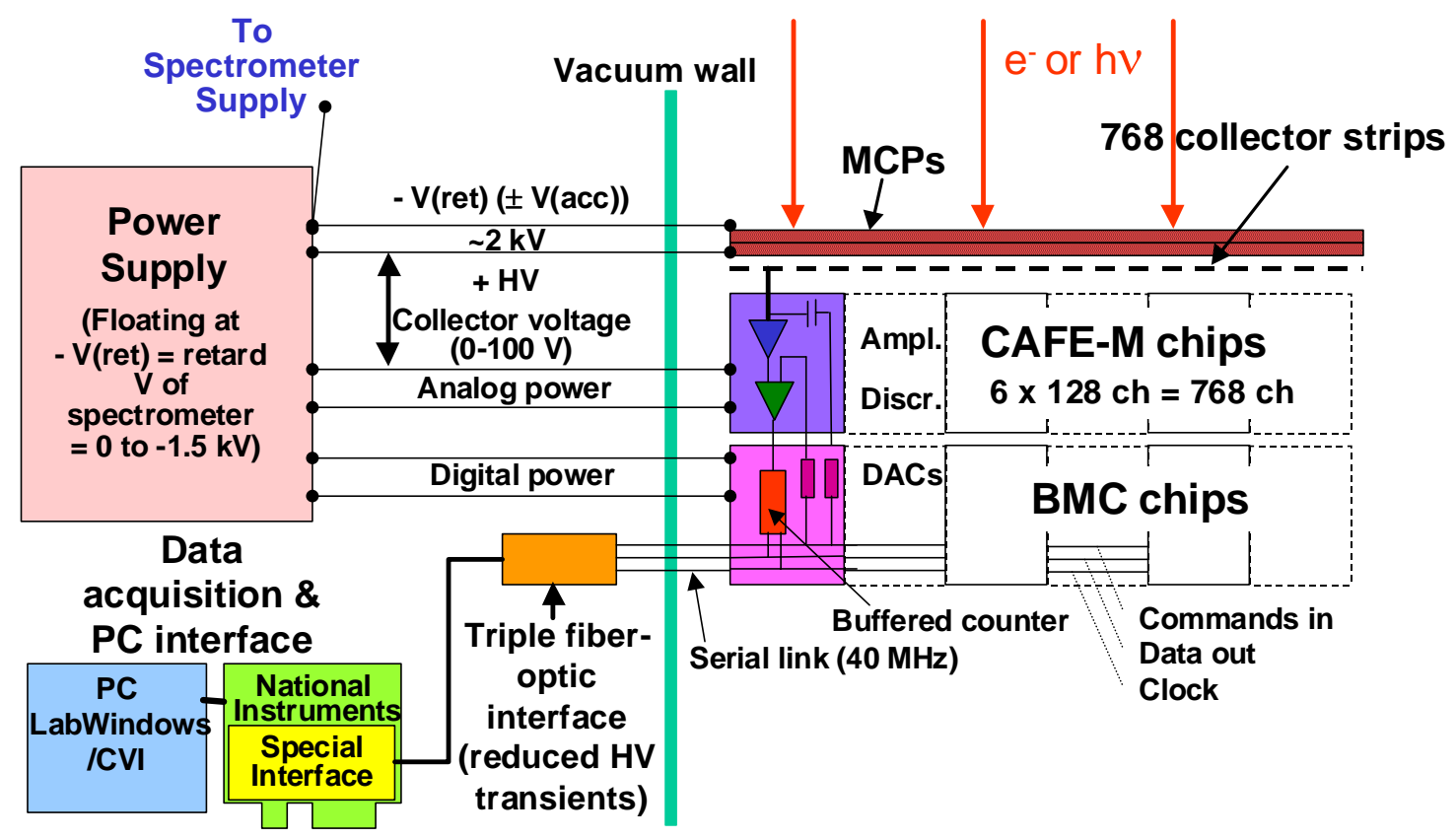

Figure 1: Ultrahigh-speed detector system schematic 
The detector is assembled on a ceramic substrate using clamps to hold the multichannel plates in place above the collector. Wirebonds connect collector strips and ICs. The assembly is surrounded by a protective shell with an outside diameter of $48 \mathrm{~mm}$ for fitting into a SES 2002 analyzer. Some ability to sense the counting distribution along the second dimension parallel to the collector electrodes will also be incorporated, via a translating slit perpendicular to the collector strips; this can be used for initial resolution optimization. A second, smaller version of the detector is being designed for use with the SES 100 analyzer or other smaller analyzers. This version will feature 512 channels connected to 2 pairs of signal processing ICs. Its active area is $25.6 \mathrm{~mm} \times 10 \mathrm{~mm}$.

The detector can float at high potential with respect to ground if needed, in particular at the retarding potential of an electron spectrometer. This float potential can be up to $2 \mathrm{keV}$, or with some special additional insulation, up to $6 \mathrm{keV}$. In order to avoid high voltage damage to ICs and other components, as well as unwanted noise via ground loops, the serial command and data lines are connected to the data acquisition system via three optical fibers. The detector is thus protected against high voltage failures such as a short from detector common to ground. A special field programmable gate array (FPGA)-based interface residing on the PC-side of the opto-interface board serializes and parallelizes the commands and reads out data, respectively. A National Instruments board is used as the PC interface. The system is operated with LabWindows/CVI-based software.

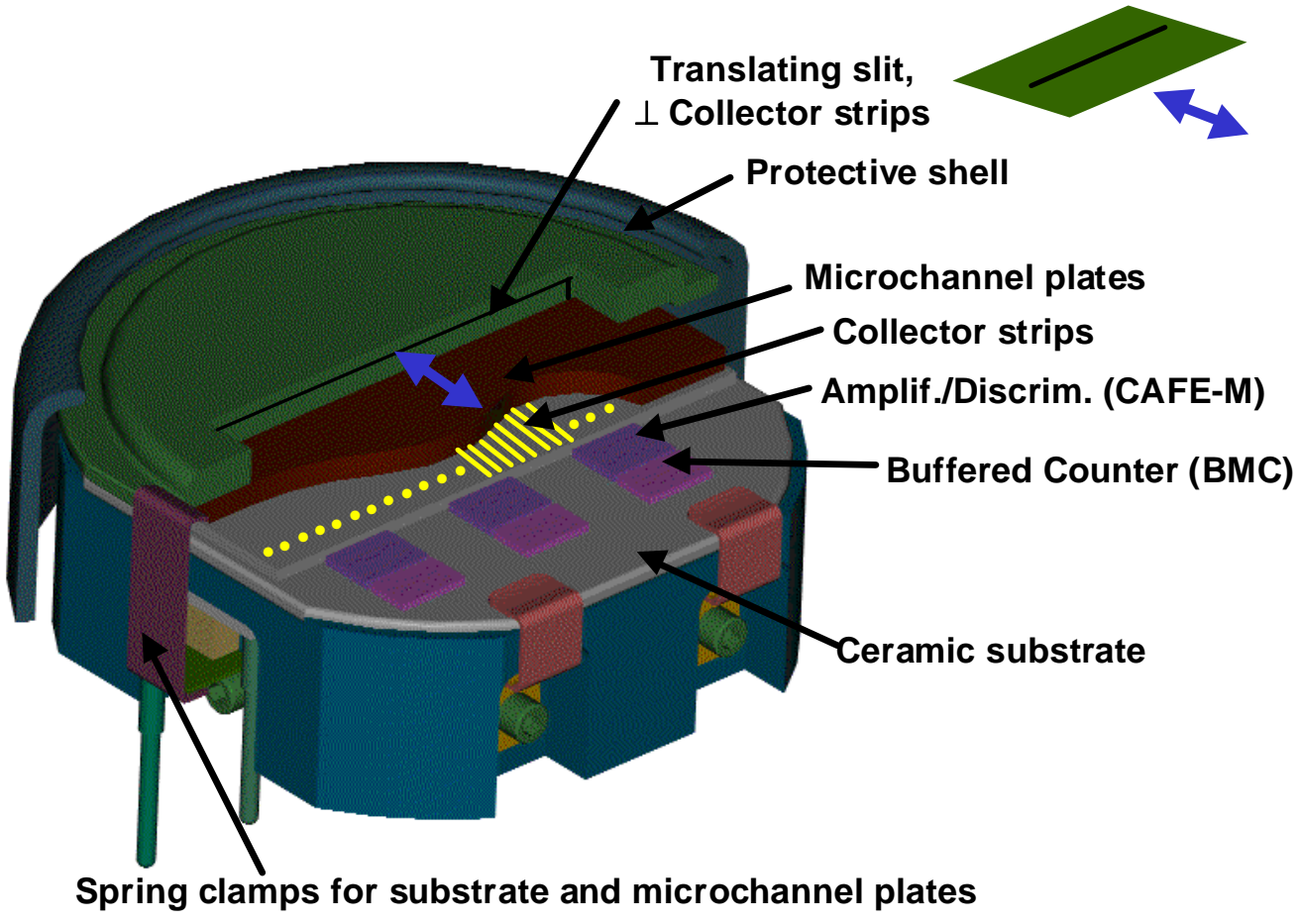

This detector will thus be able to count approximately 100 times faster than any other present detector, with significantly improved spatial resolution of 75 microns as well. The data transfer speed will exceed $40 \mathrm{MHz}$, and can be as high as $80 \mathrm{MHz}$. The number of bits to be read out from each counter is programmable. For fastest readout a small number of bits can be chosen 
making it possible to complete a spectral readout in $\sim 100 \mu \mathrm{s}$ for $40 \mathrm{MHz}$ operation, or $\sim 50 \mu \mathrm{s}$ for $80 \mathrm{MHz}$ operation.

A previous prototype of this detector, which was outfitted with less capable readout ICs, has already demonstrated the ability to count linearly at up to $1.0 \mathrm{GHz}$ overall, to resolve channels with a FWHM of 75 microns, and to operate in a typical UHV ALS environment (see attached contribution to the ALS Compendium of Abstracts for 2001).

The timeline for this project calls for a first detector to be mounted in an SES 200 electron spectrometer in Fall, 2002. Others will be fabricated once this system is tested and certified.

Overall Detector Design parameters and features:

Active detector area: $\quad 38.4 \mathrm{~mm}$ x $10 \mathrm{~mm}$ (second at $25.6 \mathrm{~mm} \mathrm{x} 10 \mathrm{~mm}$ )

Number of channels: $\quad 768$ (second at 512)

Channel pitch: $\quad 50 \mu \mathrm{m}$

Actual spatial resolution in 1D $\quad 75 \mu \mathrm{m}$ FWHM

Maximum linear countrate/channel: $\quad 2 \mathrm{MHz}$

Maximum tota linear countrate for detector: $2 \mathrm{GHz}$ (second at $1.3 \mathrm{GHz}$ )

Buffered 24 bit counter for near deadtimeless readout

Selectable (programmable) number of bits for fast readout: 4 to 24 bits

Readout time: $\quad 60 \mu$ s to $460 \mu \mathrm{s}$

Overall detector size: $\quad 65$ mm outside diameter for SES 2002

or second at $48 \mathrm{~mm}$ outside diameter for smaller analyzers

LBNL can build (under a work-for-others contract) a limited number of complete systems for collaborating laboratories that would include the detector module, the fiberoptic link, the computer interface, software for control and data acquisition, and the power supply system. The detector will be fully tested, and will come with a complete set of documentation and an instruction manual for its operation. In-house training of personnel via collaboration with LBNL scientists could also be arranged.

Cost of a complete detector system: $\$ 160 \mathrm{~K}$

Delivery: Within 9 months (6 months fabrication and 3 months for testing)

Additional information can be obtained from:

1. Bernhard Ludewigt, Staff Scientist: Bernhard_Ludewigt@lbl.gov

2. Zahid Hussain, Staff Scientist: ZHussain@lbl.gov

3. Chuck Fadley, Faculty Staff Scientist: fadley@lbl.gov

Attachment-Contribution to the ALS Compendium of Abstracts for 2001 


\title{
(Contribution to the Advanced Light Source Compendium of Abstracts, 2001)
}

\section{Development of a GHz-Rate Detector for Synchrotron Radiation Research}

\author{
B. Turko ${ }^{3}$, M. Press ${ }^{3}$, A.W. Kay ${ }^{1,2, \#, \text {,. M. West }}{ }^{1,3}$, J.E. Katz ${ }^{3}$, H. Spieler ${ }^{5}$, Z. Hussain ${ }^{4}$, \\ C.S. Fadley ${ }^{1,2}$, B. Ludewigt ${ }^{3}$, J.-M. Bussat ${ }^{3}$, P. Denes ${ }^{3}$, H. von der Lippe ${ }^{3}$, G. Meddeler ${ }^{3}$, \\ G. Zizka ${ }^{3}, \mathrm{G}$, Lebedev $^{4}$, M. Mellon ${ }^{6}$, T. Wiell ${ }^{7}$ \\ ${ }^{1}$ Materials Sciences Division, LBNL, Berkeley, CA 94720 \\ ${ }^{2}$ Dept. of Physics, UC Davis, Davis, CA 95616 \\ ${ }^{3}$ Engineering Sciences Division, LBNL \\ ${ }^{4}$ Advanced Light Source, LBNL, Berkeley, CA 94720 \\ ${ }^{5}$ Physics Division, LBNL, Berkeley, CA 94720 \\ ${ }^{6}$ Quantar Technology, Santa Cruz, CA \\ ${ }^{7}$ Gammadata/Scienta AB, Uppsala, Sweden \\ ${ }^{*}$ Present address: Intel Corporation, Portland, OR
}

\section{INTRODUCTION}

It has by now become obvious that the brightness of third-generation synchrotron radiation sources often exceeds the capabilities of the end-station detector systems to adequately handle the electron or photon fluxes resulting from a given experiment, thus preventing both the fullest utilization of the radiation and the carrying out of certain new types of experiments readily, if at all. Detector non-linearity is one problem that has been encountered [1], but there are many other examples for which beamline or spectrometer throughput must be decreased to prevent high countrate saturation, or the number of energy or angular channels that can be counted simultaneously severely limits a given experiment [2]. In recognition of this, a national-level initiative in detector development has been proposed by the multi-institutional "DetectorSync" group [2].

As a first example of what can be accomplished with advanced detector technology, a project to develop an ultrahigh-speed one-dimensional detector for electrons and vuv/x-ray photons is underway at the ALS.

\section{DESIGN PHILOSOPHY AND FIRST TEST RESULTS}

This project takes advantage of unique expertise at LBNL for detector development in high-energy and nuclear physics, and involves the custom design and fabrication of application-specific integrated circuits (ASICs). The final goal is a 768-channel detector with 50 micron spacing between channels and a maximum linear countrate per channel of over 2 megaherz. The overall countrate will thus be in the $2 \mathrm{GHz}$ range, and approximately 100 times faster than any other present one-dimensional or two-dimensional detector, with significantly improved spatial resolution as well compared to other existing detectors. First applications will be in electron spectroscopy, but others in x-ray absorption and x-ray emission spectroscopy are expected to follow.

A first prototype of this detector is shown in Fig. 1(a). Based on 12 pairs of 64-channel ASICs (an existing high-energy preamplifier chip (SDC) and a specially-designed buffered counter (DBC)), this detector has already demonstrated the ability to take spectra in a Scienta electron spectrometer located at the ALS (Fig. 2(a)), to resolve channels with a FWHM of 75 microns (Fig. 2(b)), and to count linearly at up to $1.0 \mathrm{GHz}$ overall (Fig. 2(c)) [3].

Based on this experience, a next-generation detector with significant improvements in all elements from power supplies to ASICs to data acquisition is presently under development, with an expected completion date of late 2002. This will use 6 pairs of 128-channel ASICs (a newer high-energy preamplifier chip (CAFÉ-M) and a specially-designed buffer counter (BMC)), with optical coupling between detector and control/counting electronics to minimize noise and transients. The completion date for this project is estimated to be late 2002 .

\section{CONCLUSIONS}

A prototype one-dimensional electron and photon detector operating linearly up to the $\mathrm{GHz}$ countrate level and with a resolution of 75 microns over 768 channels has been successfully developed and tested. An improved version of this detector is under development, with the same 
resolution and number of channels, but improved performance, programmability, and robustness in general user environments relative to the prototype. The next-generation detector should find use in several areas of synchrotron radiation research.

\section{REFERENCES}

[1] A.W. Kay, F.J. Garcia de Abajo, S.-H. Yang, E. Arenholz, B.S. Mun, N. Mannella, Z. Hussain, M.A. Van Hove, and C.S. Fadley, Phys. Rev. B 63, 115119 (2001); D. Nordlund, M.G. Garnier, N. Witkowski, R. Denecke, A. Nilsson, M. Nagasono, N. Martensson, A. Fohlisch, Phys. Rev. B $\underline{63}, 121402$ (2001); and abstract in the 2001 Compendium by N. Mannella et al.

[2] Local participants in the DetectorSync initiative include A. Thompson, H.A. Padmore, and C.S. Fadley, and the group's website is at--http://wwwesg.lbl.gov/esg/meetings/detectorsync/index.html, with a more detailed white paper on detector needs also downloadable from this source.

[3] A.W. Kay, Ph.D. thesis (University of California, Davis, September, 2000), LBNL report 46885, and to be published.

[4] H. Kipnis, H. Spieler and T. Collins, A Bipolar Analog Front-End Integrated Circuit for the SDC Silicon Tracker, IEEE Trans. Nucl. Sci. NS-41/4 (1994) 1095-1103

[5] Ciocio et al., A Binary Readout System for Silicon Strip Detectors at the LHC, Proceedings of the First Workshop on Electronics for LHC Experiments, Lisbon, September 11-15, 1995, CERN/LHCC/95-56, 108-113

[6] . Albiol et al., Performance of the ATLAS Silicon Strip Modules, Nucl. Instr. and Meth. A403 (1998) 247-255.

[7] T. Dubbs et al., The Development of the CAFE-P/CAFE-M Bipolar Chips for the ATLAS Semiconductor Tracker, Proc. 5th Workshop on Electronics for the LHC Experiments (LEB 99), Snowmass, Colorado, 20-24 Sep 1999, 123-127.

This work was supported by the U.S. Department of Energy, Office of Science, Office of Basic Energy Sciences, Materials Sciences Division, under Contract No. DE-AC03-76SF00098.

Principal investigator: Charles S. Fadley, Department of Physics UC Davis, and Materials Sciences Division, Lawrence Berkeley National Laboratory. Email: fadley@lbl.gov. Telephone: 510-486-5774 


\section{ALS GHz-RATE DETECTOR PROJECT}

- (a) Prototype:

- 768 channels (64 x 12 chip pairs)

- Operation in real ALS environment

- 75 micron spatial resolution

- $1 \mathrm{GHz}$ overall linear countrate

- demonstration of principle

- (b) Next generation:

- 768 channels (128 x 6 chip pairs)

- $\sim 75$ micron spatial resolution

- >2 GHz overall linear countrate

- spectral readout in as little as $60 \mu \mathrm{s}$ (time-resolved measurements)

- programmable thresholds, readout format

- more robust in all respects

- size to fit current spectrometers
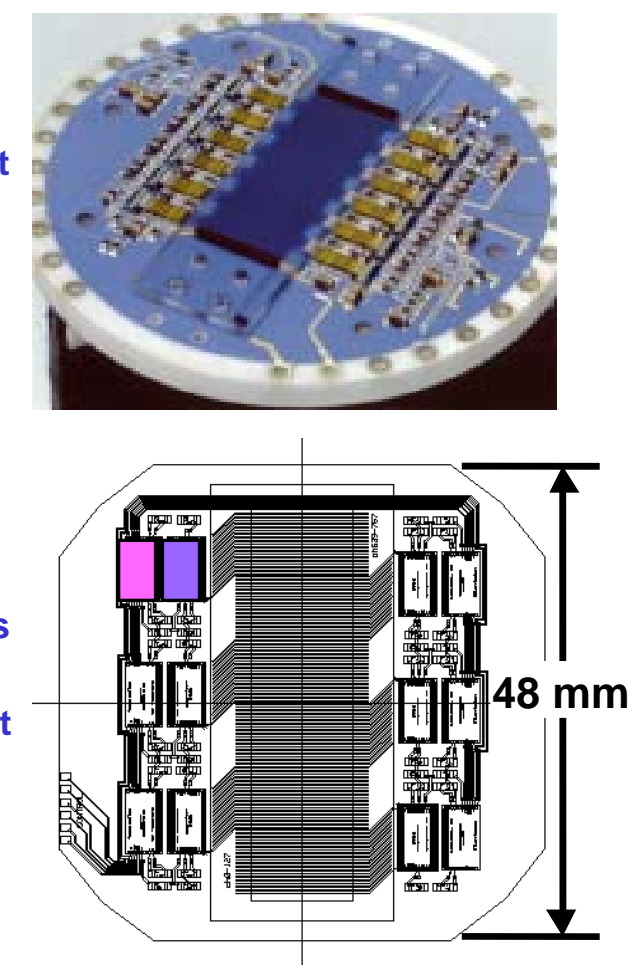

Figure 1--(a) Basic characteristics of the prototype GHz-rate detector developed as the first stage of this project, together with a photo of the ceramic substrate with 12 preamp-plus-counter chipsets mounted on it. The microchannel plates are not yet installed. (b) Basic characteristics of the next-generation detection now being designed and fabricated, together with a layour of its 6 chipsets and an indication of its size. 
(a)

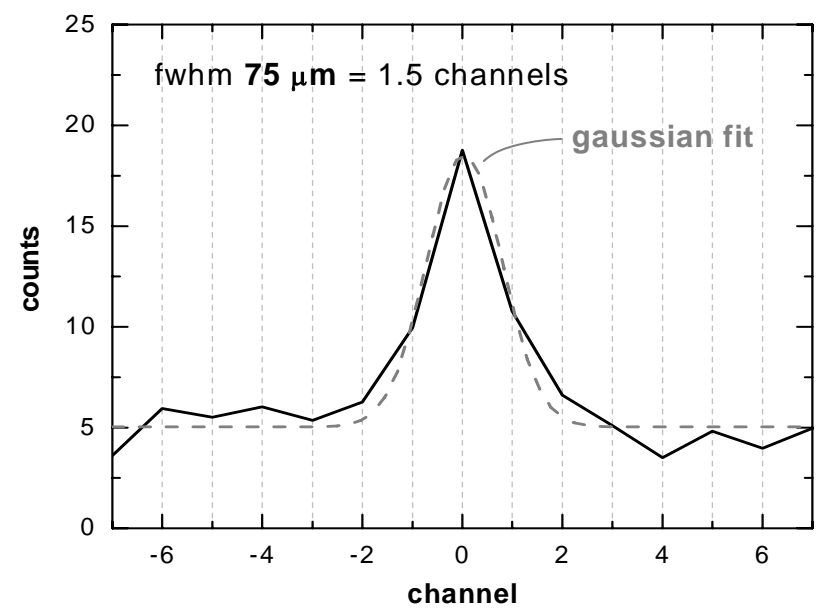

(b)

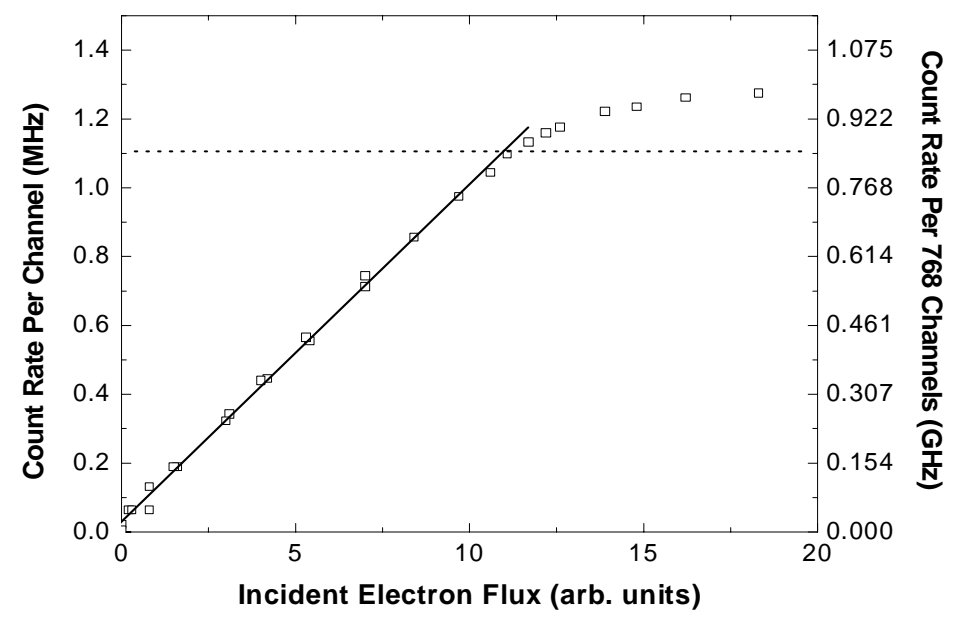

(c)

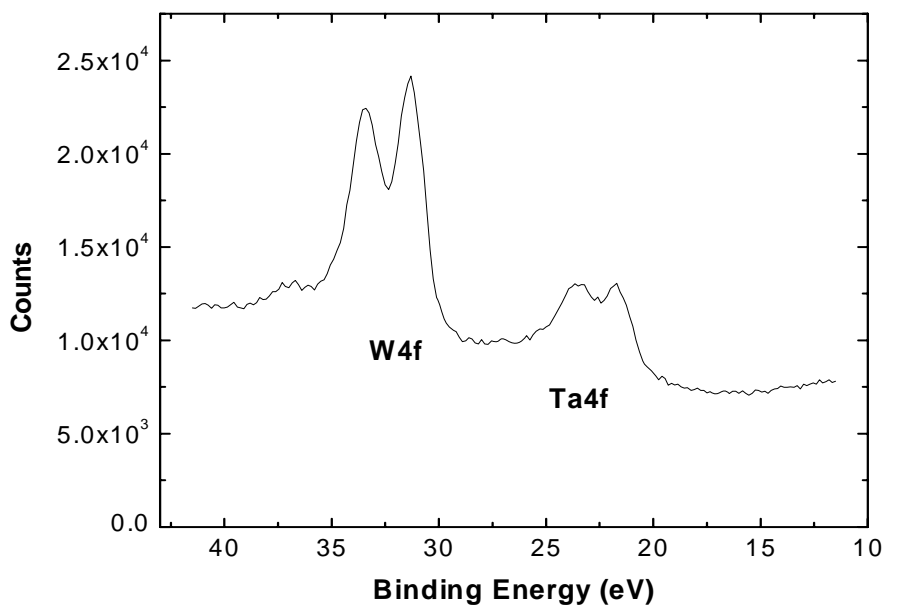

Figure 2--Test data from the first prototype detector shown in Fig. 1(a). (a) Spatial resolution determination via a collimated electron beam source. Each channel is 50 microns. (b) Counting linearity per channel (left scale) and over all channels (right scale) as determined with an electron gun. (c) Test spectra obtained with the detector mounted in a Scienta SES 200 spectrometer at ALS beamline 9.3.2. 